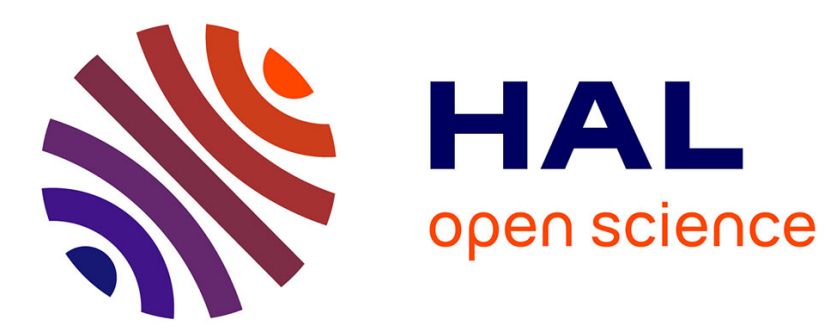

\title{
Fracture of a viscous liquid
}

Frédéric Restagno, Elise Lorenceau, David Quéré

\section{To cite this version:}

Frédéric Restagno, Elise Lorenceau, David Quéré. Fracture of a viscous liquid. Physical Review Letters, 2003, 90 (18), pp.184501. 10.1103/PhysRevLett.90.184501 . hal-00000276

\section{HAL Id: hal-00000276 https://hal.science/hal-00000276}

Submitted on 2 Apr 2003

HAL is a multi-disciplinary open access archive for the deposit and dissemination of scientific research documents, whether they are published or not. The documents may come from teaching and research institutions in France or abroad, or from public or private research centers.
L'archive ouverte pluridisciplinaire HAL, est destinée au dépôt et à la diffusion de documents scientifiques de niveau recherche, publiés ou non, émanant des établissements d'enseignement et de recherche français ou étrangers, des laboratoires publics ou privés. 


\title{
Fracture of a viscous liquid
}

\author{
Élise Lorenceau, Frédéric Restagno, and David Quéré \\ Physique de la Matière Condensée, \\ UMR 7125 du CNRS, Collège de France, \\ 75231 Paris Cedex 05, France.
}

(Dated: April 2, 2003)

\begin{abstract}
When a viscous liquid hits a pool of liquid of same nature, the impact region is hollowed by the shock. Its bottom becomes extremely sharp if increasing the impact velocity, and we report that the curvature at that place increases exponentially with the flow velocity, in agreement with a theory by Jeong and Moffatt. Such a law defines a characteristic velocity for the collapse of the tip, which explains both the cusp-like shape of this region, and the instability of the cusp if increasing (slightly) the impact velocity. Then, a film of the upper phase is entrained inside the pool. We characterize the critical velocity of entrainment of this phase and compare our results with recent predictions by Eggers.
\end{abstract}

PACS numbers: 47.15.Rq, 47.15.Gf, 47.55.Dz

We all observed that diving in a swimming-pool produces air bubbles, while entering slowly in the same pool does not: the motion of a solid penetrating a liquid at a high speed largely deforms the surface of this liquid, and induces air entrainment. This phenomenon often defines the maximum speed of coating of a solid, which enters a bath before leaving it coated. Generally air entrainment must be avoided because of the resulting bubbles (which are all the more harmful since the coating solution generally contains surfactants, and thus is likely to transform into a foam). Different experiments using either fibers or plates as solids indeed prove the existence of a threshold (in velocity) for air entrainment [1], but there is today no theoretical picture for understanding quantitatively this phenomenon.

Quite similarly, when a viscous liquid enters a pool of same nature (as it occurs for example for the stem wave of a moving ship), air entrainment may occur (producing bubbles, which ultimately burst - a serious cause of noise for a ship). Such a bubble production was observed for a liquid jet impinging a bath of same nature, as reported by Lin and Donelly, who studied experimentally the minimum velocity of air entrainment as a function of different characteristics of the jet (diameter, viscosity or surface tension) [2]. This question is important in many industrial processes where a viscous liquid (typically molten glass, metal or polymer) is poured inside a mould - and there again, the formation of bubbles is detrimental. A remarkable geometry for studying this phenomenon, and more generally the phenomenology of the regimes of deformation of the liquid bath, was proposed by Joseph [3, 国. Two counter-rotative cylinders are partially immersed in a bath, whose upper surface is observed. At a low speed of rotation, the interface between the cylinders is hardly deformed, but increasing this speed leads to the formation of a cusp-like shape. This device thus produces a singularity at a free interface, and more generally the observation and description of similar singularities has been a very active field for the past years, either theoretically [5] or experimentally - let us quote for example selective withdrawal [6], the pinch-off of a jet [7], drops running-down on a tilted solid [8] or jet eruptions [9].

Surface tension naturally opposes the formation of such singularities at a liquid/fluid interface, and for the problem of the cusp between two counter-rotative cylinders, Jeong and Moffatt calculated in a classical paper the (non-zero) radius $r$ of the tip, in the limit of small Reynolds numbers [ []] They derived an analytical expression, which can be summarized using a physical argument proposed by Hinch [5]. In this two-dimensional geometry, the tip can be seen as a thin (hemi-)cylinder, of radius $r$. The viscous drag (per unit length) on a cylinder scales as $\eta V / \ln (d / r)$, denoting $d$ as an external length, $\eta$ as the liquid viscosity and $V$ as the flow velocity around the cylinder. On the other hand, the surface tension $\gamma$ draws this cylinder (as it does on the edge of a free sheet of liquid) with a force $2 \gamma$ (there again, per unit length). Balancing both these forces yields as a tip radius:

$$
r \sim d \exp (-C a)
$$

where $C a$ is the capillary number $(C a=\eta V / \gamma)$.

This law first helps to understand why a tip forms around a well-defined velocity: the cusp becomes very sharp when $V$ is of the order of $\gamma / \eta$, a logical quantity since both these parameters play antagonist roles in the formation of the tip. But it also predicts that $r$ essentially vanishes above this velocity, and Eggers pointed out that this should lead to the crack of the tip, because of the lubrication pressure of the upper fluid, forced to flow in this very confined region 10 . He showed that beyond a critical radius for the tip, the pressure in the cusp breaks its stationary shape. Thus, this critical radius $r_{c}$ must increase with the viscosity $\eta_{0}$ of the upper fluid, and Eggers found that $r_{c}$ should scale as $\left(\eta_{0} / \eta\right)^{4 / 3}$ [10]. Together with Moffatt's result (Eq. 1), this yields as a threshold velocity $V_{c}$ of entrainment of the upper phase:

$$
V_{c} \sim \frac{\gamma}{\eta} \ln \left(\eta / \eta_{0}\right)
$$


We could have expected (naively) that entrainment of the upper fluid would occur when the viscous forces overcome capillary ones (i.e. $V_{c} \sim \gamma / \eta$ ). Eggers' theory (Eq. 2) shows that taking into account the flow of the upper phase in the cusp should induce a logarithmic correction to this simple statement. As a consequence, the threshold velocity should depend on the viscosity of the upper phase, i.e. on its nature. This might sound a posteriori quite logical, but remarkably, this dependence is expected to be extremely weak.

Here, we investigate experimentally the different situations related to this problem. We first describe the macroscopic deformation of the interface, due to the incoming flow of liquid. Then, we focus on the smaller scale of the tip. Finally, we characterize the condition of entrainment of the upper phase.

We used a horizontal cylinder of radius $R=2 \mathrm{~cm}$, halfimmersed in a bath of glycerol (or in some cases silicone oil), of density $\rho$ and viscosity $\eta$ around 1 Pa.s (Figure 1). The whole device was set in a transparent tank, and the upper fluid was either air or light oil (of density $\rho_{0}<\rho$ and viscosity $\left.\eta_{0} \ll \eta\right)$. The interfacial tension between the two fluids, denoted as $\gamma$, was measured for each experiment. The cylinder could be rotated at a controlled velocity $\Omega$, and the motion filmed with a digital camera.

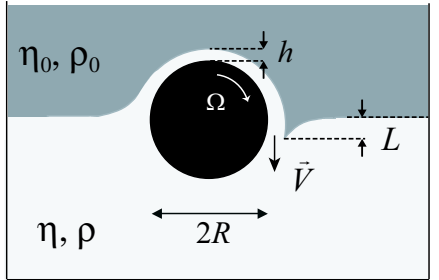

FIG. 1: Experimental set-up. A solid cylinder is placed at the interface between a viscous dense liquid and a lighter fluid. The deformation of the interface between both fluids is monitored as a function of the speed of rotation of the cylinder.

A viscous liquid film of thickness $h$ (of typically $5 \mathrm{~mm}$, thus thick enough to be independant of the nature of the roller surface) is dragged out of the bath. This layer impinges the bath at a velocity $V \simeq \Omega(R+h)$, and deforms the free surface at this place by a depth $L$. We denote $r$ as the radius of curvature of the bottom of this hollow region. Figure 2 shows the different shapes obtained with a silicon oil ( $\eta=0.97$ Pa.s), and increasing the speed of the cylinder.

It is observed that both the characteristic lengths $L$ and $r$ are dynamic in essence: the depth of immersion $L$ increases with the speed of cylinder, while the tip radius $r$ decreases. Moreover, these lengths are very different from each other: while the depth of immersion is around $1 \mathrm{~cm}$, the tip has a radius of curvature much smaller than $1 \mathrm{~mm}$. This radius can become very small when the speed is large, as first reported by Joseph et al. [3] in a similar situation. But we do not observe as they did a clear threshold velocity for the formation of a cusp - both
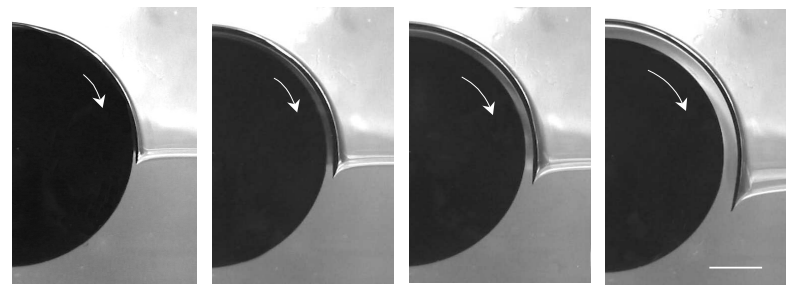

FIG. 2: Four snapshots showing the bath deformation for an increasing speed of rotation of the cylinder $(V$ is $6 \mathrm{~cm} / \mathrm{s}$, $10 \mathrm{~cm} / \mathrm{s}, 17 \mathrm{~cm} / \mathrm{s}$ and $30 \mathrm{~cm} / \mathrm{s}$, going from left to right). The lower liquid is a silicon oil of viscosity $0.97 \mathrm{~Pa}$.s and the upper fluid is air. The bar in the last picture indicates $1 \mathrm{~cm}$.

the lengths $L$ and $r$ varying continuously as a function of the cylinder velocity.

We first focus on the macroscopic length $L$, namely the depth of immersion of the tip into the bath, and report in Fig. 3 the variation of $L$ as a function of the impacting velocity $V$, for a bath of silicon oil $(\eta=0.97 \mathrm{~Pa} . \mathrm{s})$.

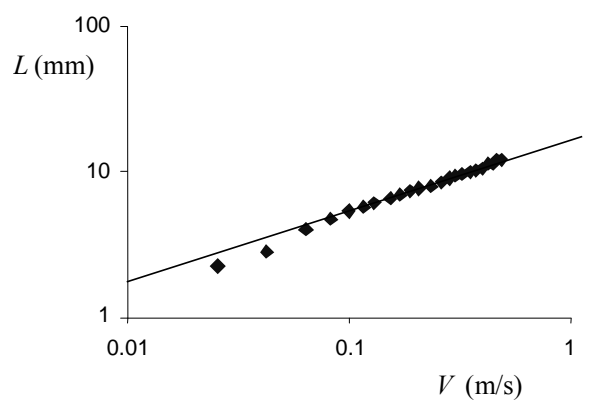

FIG. 3: Depth of immersion of the tip as a function of the velocity of the incoming liquid. The bath is a viscous silicon oil $(\eta=0.97 \mathrm{~Pa} \cdot \mathrm{s})$ with air above. The straight line indicates a slope 0.5 .

The depth $L$ of immersion of the tip is found to increase with $V$. For speeds larger than $10 \mathrm{~cm} / \mathrm{s}, L$ is several millimetres, which is significantly larger than the capillary length $\kappa^{-1}=\sqrt{\gamma / \Delta \rho g} \approx 1.5 \mathrm{~mm}$ (denoting $\Delta \rho$ as the difference of density between both fluids). We conclude that capillary forces can be neglected in this regime of large velocities. The viscous friction which pulls downward the tip can be written dimensionally $\eta V / L^{2}$, while gravitational forces per unit volume (which pull it upwards) are $\Delta \rho g$. Balancing both these forces yields:

$$
L \sim(\eta V / \Delta \rho g)^{1 / 2}
$$

Equation 3 defines some kind of viscous length (on the model of $\kappa^{-1}$ defined just above, replacing capillary effects by viscous ones). It describes fairly well the data in Figure 3, where $L$ is observed to increase as the square root of $V$. Some deviations appear at small $V$ : the deformation then is small $\left(L \sim \kappa^{-1}\right)$, and capillary effects must be considered. Since they oppose the formation 
of the hollow region, they indeed make $L$ smaller than predicted by Eq. 3 .

We then focused on the small scale of the deformation, namely the tip itself, and took macrophotographs of this region. A numerical camera was placed behind an optical microscope lighted by a classical white lamp, and the whole device first calibrated using micrometric fibers. The tip was observed to be slightly deeper close to the container walls (which allowed us to make conveniently our measurements), but remains far from it (about 15 $\mathrm{mm}$ because of the lubrification of the wall. As reported in Figure 1, the tip has a typical radius smaller than $100 \mu \mathrm{m}$, which decreases strongly with the flow velocity.
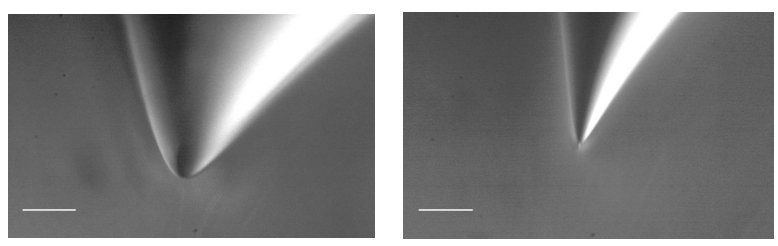

FIG. 4: Macrophotograph of the tip region (the bar indicates $200 \mu \mathrm{m}$ ), for a tip of air in a bath of glycerol. The flow velocities are respectively $14 \mathrm{~cm} / \mathrm{s}$ and $22 \mathrm{~cm} / \mathrm{s}$, and the sharpness of the tip is observed to be highly dependant on this velocity.

Analyzing the contour of the interface close to the tip allows a determination of the curvature: we can fit this contour by a parabolic function $y=a x^{2}$, from which we deduce the curvature $r=1 / 2 a$. We display in Figure 5 the measured tip radii as a function of the capillary number $C a=\eta V / \gamma$ for air and oil tips formed in a bath of glycerol.

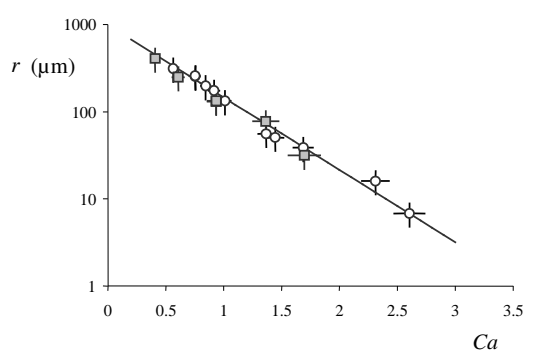

FIG. 5: Tip radius as a function of the capillary number $C a=\eta V / \gamma$ for air tips formed in a bath of glycerol (circles, $\eta=0.91 \mathrm{~Pa} \cdot \mathrm{s}, \gamma=55 \mathrm{mN} / \mathrm{m}$ ), and oil tips in a bath of glycerol (squares, $\eta=0.53 \mathrm{~Pa} \cdot \mathrm{s}, \eta_{0}=0.5 \mathrm{mPa} \cdot \mathrm{s}, \gamma=22 \mathrm{mN} / \mathrm{m}$ ). The data are deduced from macrophotographs such as the ones in Figure 1 . The plot is semi-logarithmic, and the equation of the straight fitting line is $r=993 \exp (-1.91 C a)$ (expressed in micrometers).

The tip radius is found to decrease in a continuous way, as a function of the flow velocity $V$. More precisely, its logarithm varies linearly with $V$ - in agreement with Moffatt's law (Eq. 11), on nearly two orders of magnitude for the tip radius. This exponential behavior helps understanding why the cusp-like shape apparently sets for a well-defined velocity, of the order of $\gamma / \eta$, although the actual variation of the radius is continuous with $V$. But it also suggests that slightly above this velocity, the cusp should become extremely sharp. This is not the case: a small increase of the roller velocity leads to the destruction of the tip, and the upper phase then is entrained, as reported in Figure 6. Note that the entrainment occurs simultaneously all along the tip, showing the $2 \mathrm{D}$ nature of the phenomenon.

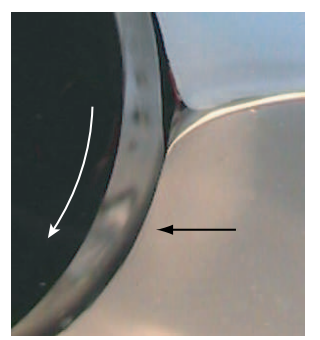

FIG. 6: Above a well-defined velocity $V_{c}$, a thin yet visible sheet of the upper fluid (here air), appearing in black in the image and stressed by an arrow, comes out of the tip and enters the viscous bath (here glycerol). The velocity $V$ is $32 \mathrm{~cm} / \mathrm{s}$, while the threshold $V_{c}$ for air entrainment is $30 \mathrm{~cm} / \mathrm{s}$.

We denote $V_{c}$ as the threshold velocity and $C a_{c}$ as the corresponding capillary number. Our experiments consisted in determining $C a_{c}$. Firstly, we varied the bath viscosity $\eta$, using mixtures of water and glycerol and air as the upper fluid. $\eta$ was measured for each solution. The corresponding data are displayed in black in Figure 7 . Secondly, we used pure glycerol for the bath, and various light oils (such as hexane, hexadecane and silicone oils) as the upper phase. We measured $\gamma$ for each oil (it was found to be quite constant, around $26 \pm 1 \mathrm{mN} / \mathrm{m}$ ). The corresponding critical capillary numbers are represented by white points, in Figure 18, where all our results are plotted as a function of the viscosity ratio $\eta_{0} / \eta$, in a semi-logarithmic scale.

We clearly observe an effect of the viscosity $\eta_{0}$ of the upper fluid (open points): the larger $\eta_{0}$, the smaller the critical capillary number. Nevertheless, this dependency is weak, since it appears to be well described by a logarithmic law. It is also limited: if the upper fluid is too viscous (typically $\eta_{0} / \eta>0.1$ ), the lower liquid is not entrained on the roller. For a given upper fluid, varying the bath viscosity $\eta$ also leads to a (weak) variation of the threshold in capillary number - which thus is not simply given by a value of order unity. All the data lie on the same semi-logarithmic plot, in agreement with Eggers' model (Eq. 2), for a variation on five orders of magnitude of the viscosity ratio $\eta_{0} / \eta$. However, the slope deduced from the fit is -0.22 , larger than -1 the value predicted by Eq. 2.

On the whole, these experiments may help clarifying the question of the stationary pattern which sets as a 


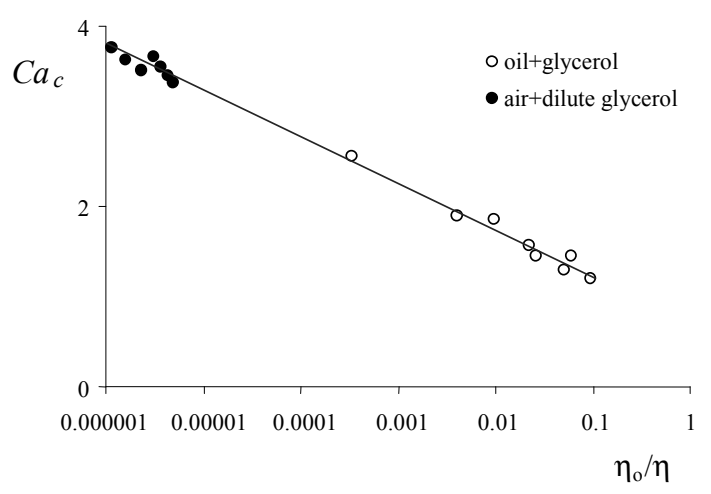

FIG. 7: Critical capillary number $C a_{c}=\eta V_{c} / \gamma$ above which the upper phase is entrained in the viscous bath as a function of the ratio between both viscosities (semi-logarithmic scale). The full points are obtained using water-glycerol mixtures $(250 \mathrm{mPa} \cdot \mathrm{s}<\eta<1500 \mathrm{mPa} \cdot \mathrm{s})$ with air above, while the open points correspond to glycerol $(\eta=900 \mathrm{mPa} \cdot \mathrm{s})$ with above different light oils $\left(0.3 \mathrm{mPa} \cdot \mathrm{s}<\eta_{0}<83 \mathrm{mPa} \cdot \mathrm{s}\right)$.

viscous liquid hits itself, in a two-dimensional geometry. If the impinging velocity is large enough (i.e. for capillary numbers typically larger than 0.1 ), two very different dynamic lengths can be defined: a global one, which is the depth of liquid hollowed by the impact, and which results from a balance between viscous effects and gravity; and a local one, at the bottom of this hollow region which can be expressed as the radius of curvature at this place. As predicted by Moffatt [5], this radius decreases exponentially with the capillary number. This law defines a characteristic velocity for which the hollow region becomes very sharp, i.e. takes a cusp-like shape, as first reported by Joseph [3]. At the same time, it predicts a collapse of the tip radius for finite velocities, which implies the crack of the tip (entrainment of the upper phase), as postulated by Moffatt [5] and shown theoretically by Eggers [10]. We indeed report here such a crack, and show that the critical velocity for which it occurs is mainly governed by the bath viscosity, but also depends (in a much weaker manner) on the viscosity of the upper phase - in agreement with Eggers' theory.
Different studies could naturally complement this one. Firstly, it would be worth understanding (both experimentally and theoretically) the thickness of the film of air, in the regime of entrainment. The selection of this thickness could remind the Landau-Levich problem (selection of the thickness of the film adhering to a solid withdrawn out of a bath), but the problem here is purely dynamical, which raises an interesting (and open) question (as in selective withdrawal, for the radius of the emitted filament). The problem of the stability of this film, which eventually breaks in bubbles, also remains to be studied. Another issue concerns the practically important question of water, for which the threshold velocity is much higher, which would require to study the influence of inertia in all the different processes described all along this study. Secondly, we observed a deviation towards the law displayed in Figure 7 using a very viscous silicone oil as a bath (instead of glycerol), and air above. Then, for $\eta>10^{5} \eta_{0}$, we measured a critical capillary number much higher, typically of the order of 5 to 10 (and increasing rapidly as decreasing the ratio $\eta_{0} / \eta$ ). A possible cause of this discrepancy could be the polymeric nature of the liquid (for which the chains are all the longer since the viscosity is high), which could lead to non-Newtonian effects close to the tip - and thus to changes in the threshold of air entrainment. Using newtonian silicone oils of smaller viscosity (with either air or ethanol above) indeed yields data in very close agreement with the ones displayed in Figure 7. Finally, it would be useful to connect this study with similar ones in other dimensionality. In particular, Cohen stressed that for selective withdrawal (i.e. one-dimensional tip forming when sucking locally a liquid above an interface), the viscosity of the fluid forming the tip does not affect the value of the cut-off curvature 11. This could be due to the fact that the Stokes law on a (hemi-)sphere does not contain any logarithmic dependency, as it does for a (hemi-)cylinder, which finally emphasizes that the effects reported here are two-dimensional in essence.

We thank Jens Eggers for stimulating discussions, Ghislaine Ducouret who helped us in the viscosity measurements and Gilles Jandeau and Daniel Détry for their technical help.
[1] R. Burley, Industrial Coating Research 2, 95 (1992).

[2] T. Lin and H. Donelly, A.I.Ch.E.J. 12, 563 (1981).

[3] D. Joseph, J. Nelson, M. Renardy, and Y. Renardy, J. Fluid Mech. 223, 383 (1991).

[4] D. Joseph, J. Fluid Mech. 44, 127 (1992).

[5] J.-T. Jeong and H. Moffat, J. Fluid Mech. 241, 1 (1992).

[6] I. Cohen and S. Nagel, Phys. Rev. Lett. 88, 074501 (2002).

[7] I. Cohen, M. Brenner, J. Eggers, and S. Nagel, Phys.
Rev. Lett. 86, 1147 (1999).

[8] T. Podgorski, J.-M. Flesselles, and L. Limat, Phys. Rev. Lett. 87, 036102 (2001).

[9] B. Zeff, B. Kleber, J. Finberg, and D. Lathrop, Nature (London) 403, 401 (2000).

[10] J. Eggers, Phys. Rev. Lett. 86, 4290 (2001).

[11] I. Cohen, Preprint Physics/0201037 (2002). 